Check for updates

Cite this: RSC Adv., 2019, 9, 37526

\title{
Theoretical investigation of various aspects of two dimensional holey boroxine, $\mathrm{B}_{3} \mathrm{O}_{3} \dagger$
}

\begin{abstract}
Saif Ullah, (D)*b Pablo A. Denis (iD)*a and Fernando Sato ${ }^{\mathrm{b}}$
By means of first-principles calculations, we study the structural, electronic and mechanical properties of the newly synthesized boron-oxygen holey framework (Chem. Comm. 2018, 54, 3971). It has a planar structure formed by $\mathrm{B}_{3} \mathrm{O}_{3}$ hexagons, which are joined via strong covalent boron-boron bonds. The six $\mathrm{B}_{3} \mathrm{O}_{3}$ units are connected with six-fold symmetry exhibiting a large hole with a surface area of $23 \AA^{2}$, which is ideal for the adsorption of alkalis. For neutral alkalis, we found that the adsorption energy of potassium is 14 and $12 \mathrm{kcal} \mathrm{mol}^{-1}$ larger than those determined for sodium and lithium, respectively. In contrast, for alkali cations, there is a clear preference for lithium over sodium and potassium. With regard to its electronic properties, it is an insulator with an electronic band gap of $5.3 \mathrm{eV}$, at the HSE level of theory. We further investigate the effect of strain on the band gap and find it a less efficient technique to tune the electronic properties. The wide optical gap of $\mathrm{B}_{3} \mathrm{O}_{3}$ can be utilized in ultraviolet (UV) applications, such as UV photodetectors, etc. Additionally, the $2 \mathrm{D}$ elastic modulus of $\mathrm{B}_{3} \mathrm{O}_{3}\left(53.9 \mathrm{~N} \mathrm{~m}^{-1}\right)$ is larger than that of $\mathrm{Be}_{3} \mathrm{~N}_{2}$, silicene, and germanene. Besides, we also report bilayer and graphite-like bulk $\mathrm{B}_{3} \mathrm{O}_{3}$ and furthermore, find that the optoelectronic properties of the bilayer can be tuned with an external electric field. The great tunability of optical properties from UV to the visible range offers a vast range of applications in optoelectronics.
\end{abstract}

Received 12th September 2019 Accepted 10th November 2019

DOI: $10.1039 / \mathrm{c} 9 \mathrm{ra} 07338 \mathrm{~h}$

rsc.li/rsc-advances along with three molecules of water. Although the boroxine layers could readily be identified in the STM studies, the authors suggested that further improvements in the synthetic procedure should be made in order to reduce the occurrence of non-six-fold symmetry motifs. Concomitantly, Lin et al. ${ }^{16}$ proposed a porous boron oxide monolayer with a robust band gap, which is similar to the one synthesized by Stredansky et al. ${ }^{15}$ They showed that boroxine is perfectly planar with uniform pores with a $6.27 \AA$ diameter and exhibit an indirect wide band gap with the possible applications in optoelectronics This work is the extension to the previous predicted (theoretical) and synthesized (experimentally) boroxine by exploring the microscopic science and including new interesting capabilities such as the high affinity for alkalis and the study of bilayer boroxine as well as the bulk layered material. As by-product, we have found a minor discrepancy in the electronic structure of boroxine. In effect, our HSEH1PBE calculations indicated that the gap is direct, in contrast with the results reported by Lin et al. ${ }^{16}$ In the text, the only " $\mathrm{B}_{3} \mathrm{O}_{3}$ " will refer to the monolayer $\mathrm{B}_{3} \mathrm{O}_{3}$ unless it is followed by "ring" or "hexagon".

\section{Methods}

${ }^{a}$ Computational Nanotechnology, DETEMA, Facultad de
11800 Montevideo, Uruguay. E-mail: pablod@fq.edu.uy

${ }^{b}$ Departamento de Física, Instituto de Ciências Exatas, Campus Universitário, Universidade Federal de Juiz de Fora, Juiz de Fora, MG 36036-900, Brazil. Fax: +55 589229241906; Tel: +55 59899714280

$\dagger$ Electronic supplementary information (ESI) available. See DOI: $10.1039 / \mathrm{c} 9 \mathrm{ra} 07338 \mathrm{~h}$
We study boroxine $\left(\mathrm{B}_{3} \mathrm{O}_{3}\right)$ by means of spin-polarized vdW-DF, ${ }^{17}$ M06-L, ${ }^{18}$ and HSEH1PBE ${ }^{19}$ density functional theory (DFT) calculation. We use SIESTA ${ }^{20,21}$ code to perform vdW-DF calculations. We select the double-zeta basis set with polarization 
functions and fixed the orbital confining cut-off to 0.01 Ry with the default value $(0.15)$ of split-norm. The interaction between ionic cores and valence electrons is described by the TroullierMartins norm-conserving (NC) pseudopotentials. ${ }^{22}$ Geometry optimizations were carried out using the conjugate gradient algorithm until all the residual forces were smaller than $0.01 \mathrm{eV}$ $\AA^{-1}$. The unit cells were optimized and they were sampled using a $50 \times 50 \times 1$ (about $900 k$-points gamma centered) Monkhorst-Pack sampling, the periodic M06-L and HSEH1PBE calculations were carried out with Gaussian $09 .{ }^{23}$ The basis set selected were Pople's 6-31G* and 6-311G*.24 In general, we did not observe significant differences in the results obtained using both basis sets. $1000 k$-points were used to sample the unit cell and the ultrafine grid was employed. For comparative calculations, we also investigate the adsorption of alkalis employing a boroxine $\left(\mathrm{B}_{3} \mathrm{O}_{3}\right)$ cluster formed by six $\mathrm{B}_{3} \mathrm{O}_{3}$ units and terminated with hydrogen atoms. In general, the results indicate the same trend as that obtained employing infinite models.

We also perform PBE-D2 (ref. 25) and PBE-D3 (ref. 26) calculations with the help of VASP code. ${ }^{27}$ We use generalized gradient approximation (GGA) with the flavor proposed by Perdew, Burke, and Ernzerhof (PBE) ${ }^{28,29}$ assisted with the projector augmented wave (PAW). ${ }^{30}$

Finally, we also use a hybrid functional (not in a true sense) which is a mixture of LDA-GGA ${ }^{28,29,31}$ as implemented in SIESTA, especially for the calculation of in-plane elastic modulus to compare our results with our previous work on graphene and $\mathrm{Be}_{3} \mathrm{~N}_{2} \cdot{ }^{32}$ The details are in ref. 31 . Throughout the manuscript, the hybrid is referring to the aforementioned method. Finally, we also report ab initio molecular dynamics (AIMD) simulations at $300-600 \mathrm{~K}$ with the aid of SIESTA code. Further details regarding the computational methods can be seen in the ESI. $\dagger$

\section{Results and discussion}

\subsection{Structural properties of $2 \mathrm{D}$ boroxine}

As suggested by the experimental study ${ }^{\mathbf{1 5}}$ and the theoretical study by Lin et al.,${ }^{16}$ we find that $2 \mathrm{D}$ boroxine can adopt six-fold symmetry. The unit cell employed in our calculations is shown in Fig. 1.

The six-fold symmetry structure is obtained by joining the $\mathrm{B}_{3} \mathrm{O}_{3}$ hexagons obtained after the condensation of three tetrahydrodiboron molecules. The B-B bonds are 1.71-1.72 A long, a value which is bracketed by the B1-B1 and B1-B2 bond distances found in Pmmn borophene, namely, 1.613 and 1.879 $\AA$, respectively, which are in excellent agreement with the PBE results previously reported. ${ }^{16}$ The $\mathrm{B}-\mathrm{O}$ bond length $1.38-1.39 \AA$ is among the highest values of which $\mathrm{B}-\mathrm{O}$ can adopt in molecular forms. As suggested by Coulson, ${ }^{32}$ this may indicate a moderate to weak participation of the $\pi$ orbitals of boron and oxygen. A fact that seems to be corroborated by the partial density of states presented in Fig. 2. When the six $\mathrm{B}_{3} \mathrm{O}_{3}$ molecules are joined, they leave a hole in which the distance between the opposite $\mathrm{O}$ atoms is 6.21-6.28 $\AA$ (depending on the level of theory) and the surface is close to $23 \AA^{2}$, ideal for the adsorption of large atoms or molecules, as observed for crown ethers. For example, the 18-crown-6 ether has a high affinity for the hydronium ion $\mathrm{H}_{3} \mathrm{O}^{+}$. In addition to this, it allows the dissolution of $\mathrm{KMnO}_{4}$ in benzene thanks to the complexation of the potassium ion. We show in Section 3.8 that 2D boroxine has also a strong affinity towards alkalis, especially to potassium.

To report the structural stability, we calculate the formation energy and since we use the atomic form of $\mathrm{B}$ and $\mathrm{O}$ as the chemical potential, the formation energy here is equal to the cohesive energy. We make use of the following expression: $E_{\text {coh }}=\frac{E_{\mathrm{B}_{3} \mathrm{O}_{3}}-6 E_{\mathrm{B}}-6 E_{\mathrm{O}}}{12}$, where $E_{\mathrm{B}_{3} \mathrm{O}_{3}}$ is the total energy of the unit cell containing $6 \mathrm{~B}$ and $6 \mathrm{O}$ atoms. $E_{\mathrm{B}}$ and $E_{\mathrm{O}}$ are taken as the gas-phase energies of $\mathrm{B}$ and $\mathrm{O}$, respectively. The cohesive energy of $\mathrm{B}_{3} \mathrm{O}_{3}$ PBE-D3 level is $-6.72 \mathrm{eV}$ per atom, just 0.04 lower than the value reported by Lin et al. ${ }^{\mathbf{1 6}}$ For the comparison, the cohesive energy of graphene at the same level of theory is $-8.07 \mathrm{eV}$ per atom. This means that $\mathrm{B}_{3} \mathrm{O}_{3}$ has a comparable strength to that of graphene. At the M06-L level, the cohesive energy of $\mathrm{B}_{3} \mathrm{O}_{3}-6.3 \mathrm{eV}$ per atom which is twice the value calculated for phosphorene $(-3.1 \mathrm{eV}$ per atom) at the same level. Further information is given in ESI. $\uparrow$ The data regarding structural properties and stability at various levels of theory is shown in Table S1.†

\subsection{Dynamical stability}

In the experiment, 2D boroxine $\left(\mathrm{B}_{3} \mathrm{O}_{3}\right)$ was grown on $\mathrm{Au}(111)$ surface and, therefore, it is necessary to check the dynamical stability of free-standing boroxine $\left(\mathrm{B}_{3} \mathrm{O}_{3}\right)$. To that end, we calculate phonon spectrum by supercell approach as implemented in SIESTA code along the G-M-K-G high symmetry points. We use a $2 \times 2 \times 1$ supercell to calculate the phonon band spectrum, whereas, a $1 \times 1 \times 1$ cell also give converged result, keeping in mind that the unit cell size is already large enough. The phonon spectrum graph is shown in Fig. 2 where it is clear that $2 \mathrm{D}$ boroxine is certainly a local minimum in the potential energy surface due to the absence of any negative frequency. The highest frequency of $\mathrm{B}_{3} \mathrm{O}_{3}$ is $\sim 1450 \mathrm{~cm}^{-1}$.

\subsection{Electronic properties}

The electronic properties of $2 \mathrm{D}$ boroxine are predicted by calculating the electronic band structure and for additional comprehension, the orbital projected density of states (PDOS) is also computed. With SIESTA (both the levels employed), 2D boroxine $\left(\mathrm{B}_{3} \mathrm{O}_{3}\right)$ is a wide and indirect band gap material with conduction band minima (CBM) lie at $\mathrm{M}$ point, while valence band maxima (VBM) at gamma point. However, the energy difference between gamma and $\mathrm{M}$ points is quite low (55 meV). This indirect gap nature is also confirmed by VASP code with a slight disagreement as the CBM appears at the $K$ point which is in line with the previous findings by Lin et al. ${ }^{16}$ The calculated value of band gap, at vdW-DF level is $3.80 \mathrm{eV}$. However, the M06$\mathrm{L}$ level of theory revealed a direct gap with a value of $3.9 \mathrm{eV}$. As expected, the band gap was significantly underestimated by the vdW-DF method and also by the M06-L functional. In effect, HSEH1PBE calculations indicated that the band gap of boroxine is direct, namely $5.30 \mathrm{eV}$ which is a bit higher than the previously reported value of $5.23 \mathrm{eV}$. The latter value is smaller than 


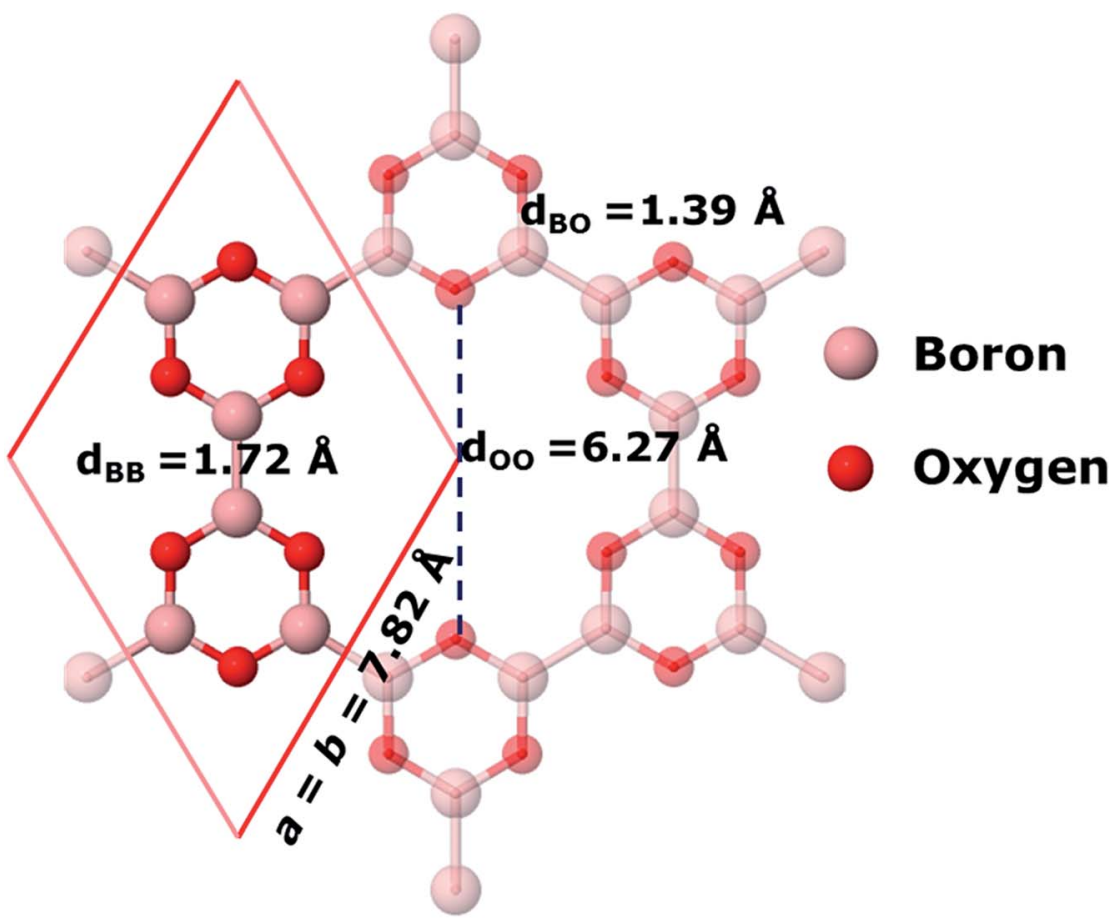

Fig. 1 The unit cell and bond lengths of $2 \mathrm{D}$ boroxine $\left(\mathrm{B}_{3} \mathrm{O}_{3}\right)$ at PBE-D3 level of theory. Periodically repeated atoms are made translucent.

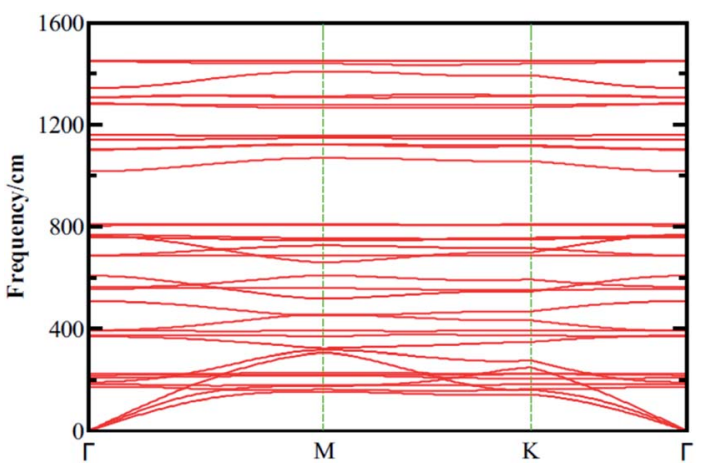

Fig. 2 Phonon dispersion curves calculated for boroxine $\left(\mathrm{B}_{3} \mathrm{O}_{3}\right)$ calculated at vaW-DF/DZP level of theory with SIESTA code.

the gap computed for boron nitride at the HSEH1PBE level, namely, $6.1 \mathrm{eV}$. Moreover, $\mathrm{B}_{3} \mathrm{O}_{3}$ bears the same direct-indirect band gap controversy as that of boron nitride.

From the PDOS, the CBM has a $\pi$ character and mostly composed of B- $\mathrm{p}_{z}$ with a slight involvement from O- $\mathrm{p}_{z}$. However, the O- $\mathrm{p}_{(x+y)}$ strongly hybridized with B- $\mathrm{p}_{(x+y)}$ to form the VBM and, therefore, has a $\sigma$-like character. Furthermore, the valence and conduction bands are mostly governed by $\mathrm{O}$ and $\mathrm{B}$, respectively. Additionally, this outcome also reflects in LDOS plots shown in Fig. 3 where the density around $\mathrm{O}$ atoms can be seen in VB case, whereas, for $\mathrm{CB}$, the density can be spotted around $\mathrm{B}$ and $\mathrm{O}$ with a larger LDOS amplitude on $\mathrm{B}$ in comparison with O. Besides, we also check the effect of strain on the electronic structure of $\mathrm{B}_{3} \mathrm{O}_{3}$ and find that it is a less efficient technique to alter the electronic properties. The band structures are given in Fig. S1 in ESI. $\dagger$

\subsection{Chemical analysis and bonding character}

We calculate the charge transfer with the help of various charge transfer mechanisms namely Hirshfeld and Voronoi as in SIESTA, Mulliken as in Gaussian, and Bader as in VASP. Each of these methods reveals qualitatively the same results of charge transfer from $\mathrm{B}$ to $\mathrm{O}$ as in the spirit of their electronegativity values. The total charge density calculated at PBE-D3 level is plotted in Fig. 4(a), where charge accumulation on $\mathrm{O}$ atoms can be seen quite vividly. The quantitative results are given in Table $\mathrm{S} 1 . \dagger$ Furthermore, to picture the electron localization in $\mathrm{B}_{3} \mathrm{O}_{3}$, we calculate electron localization function (ELF) which is quite useful in predicting the bonding character, as well as the stability mechanism. The details regarding the interpretation of ELF plots can be seen in ref. 31 and 33. In $_{\mathrm{B}_{3}} \mathrm{O}_{3}$ rings, we see electron localization on $\mathrm{O}$ atoms broadens towards $\mathrm{B}$ atoms and a complete charge delocalization in the vicinity of B atoms. This is in perfect agreement with the previous experimental + DFT study on $\mathrm{B}_{3} \mathrm{O}_{3}$ rings held together by phenyl molecules where the authors reported the ultra-fast charge delocalization with the charge localization on $\mathrm{O}$ atoms ${ }^{34}$ Such an analysis, however, is absent for phenyl-free $\mathrm{B}_{3} \mathrm{O}_{3}$ rings which are bonded via $\mathrm{B}$ atoms. Consequently, the ELF becomes crucial and much more meaningful. Interestingly, we find a strong charge localization between $\mathrm{B}-\mathrm{B}$ atoms, the region between two $\mathrm{B}_{3} \mathrm{O}_{3}$ rings. This indicates that the $\mathrm{B}_{3} \mathrm{O}_{3}$ rings are held together by strong covalent bonds with the ionic bonds (by virtue of charge transfer) present between $\mathrm{B}-\mathrm{O}$. This strong covalent bond might be responsible for the stability of $\mathrm{B}_{3} \mathrm{O}_{3}$ monolayer consisting of $\mathrm{B}_{3} \mathrm{O}_{3}$ rings. Moreover, we also calculate simulated scanning tunneling microscopy (STM) image (based on Tersoff-Hamann 

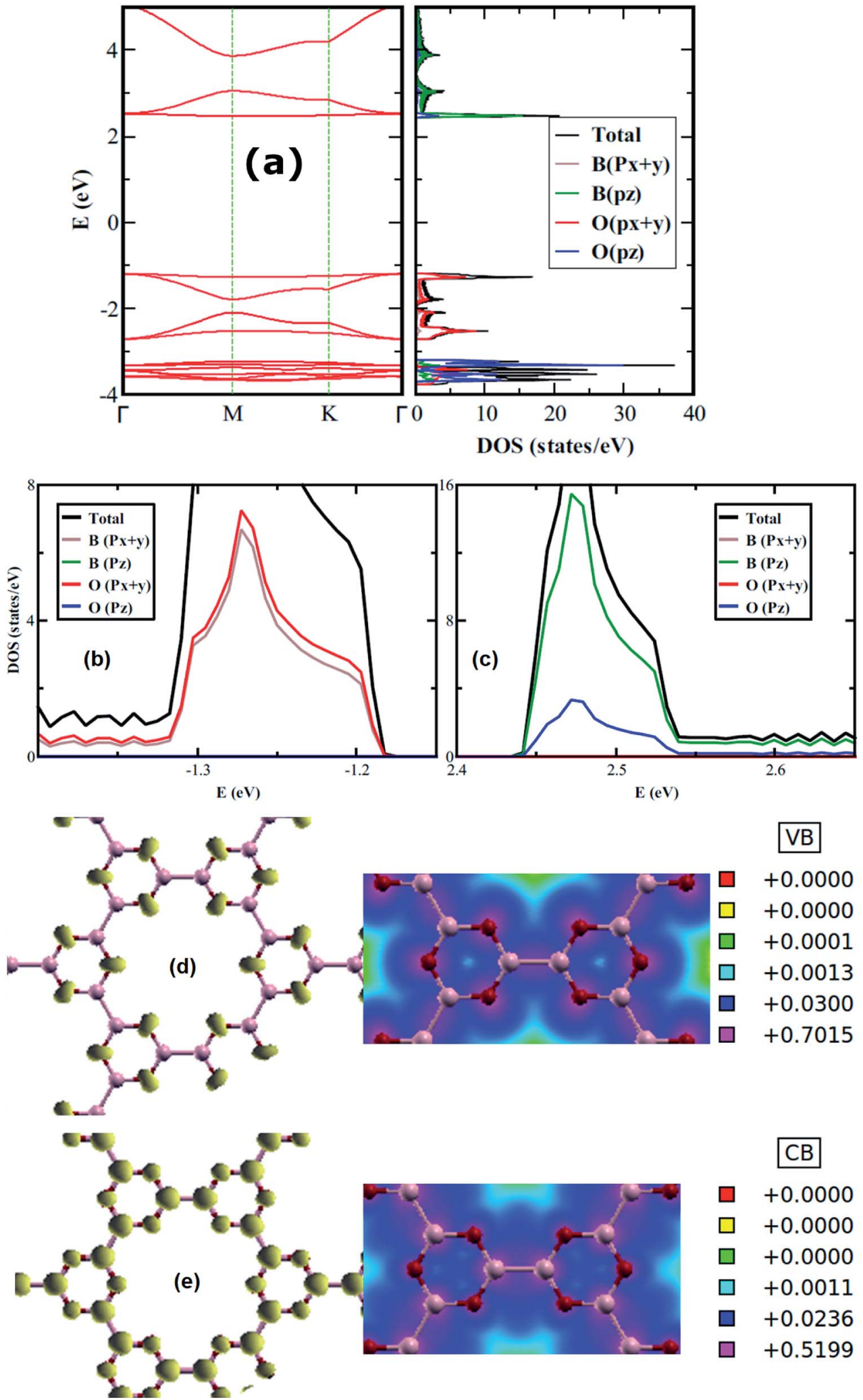

VB

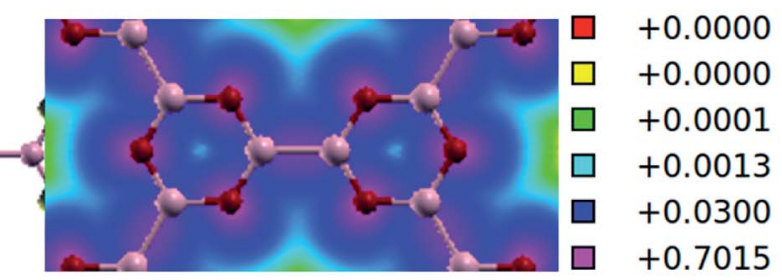

Fig. 3 Electronic band structure along with (total and projected) density of states (a); projected density of states near the (b) valence band maximum and (c) the conduction band minimum; 3D plots along with their 2D cuts of LDOS of filled (d) and empty states (e) of boroxine calculated at the hybrid level of theory. 


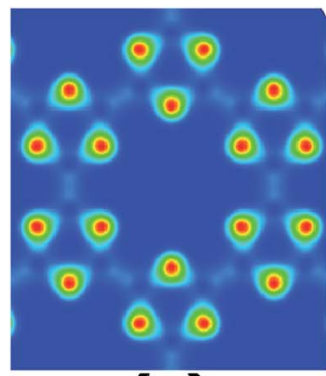

(a)

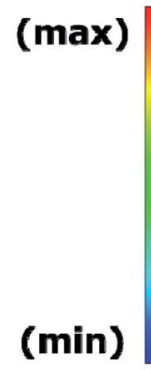

(min)

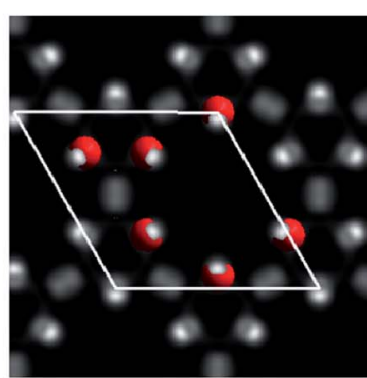

(d)

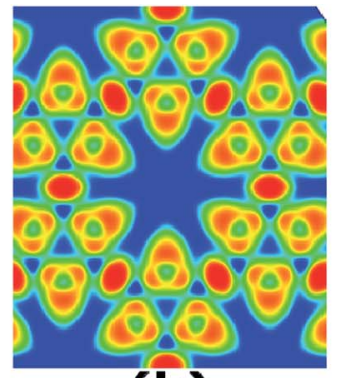

(b)

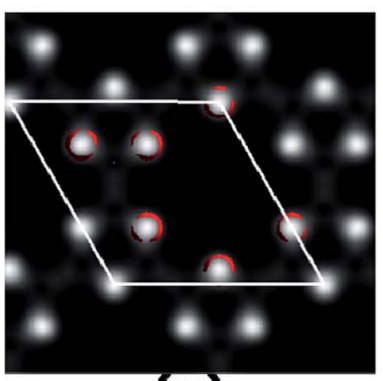

(c) represents a region with a homogenous electron density, (c) STM image of $\mathrm{B}_{3} \mathrm{O}_{3}$, (d) STM image of filled and (e) empty states.

approximations) ${ }^{35}$ of $\mathrm{B}_{3} \mathrm{O}_{3}$ monolayer which is given in (c) and is in agreement with the experimental result. $\mathrm{O}$ atoms in the unit cell are shown for the sake of brevity. In addition, we also calculate STM images of the filled and empty states which are in agreement with the PDOS (and LDOS) calculations.

\subsection{Kinetic stability}

We also test boroxine with high temperatures (300 and $600 \mathrm{~K}$ ) to see the response. The initial and final structures treated at $600 \mathrm{~K}$ are shown in Fig. 5. The negligible structural modifications suggest that $\mathrm{B}_{3} \mathrm{O}_{3}$ is quite stable at high temperatures. This point has already been proved experimentally. In addition, we also calculate the average change appeared in the $\mathrm{B}-\mathrm{O}$ and $\mathrm{B}-\mathrm{B}$ bond lengths at a temperature of $600 \mathrm{~K}$. We find a slight variation of $0.986 \%$ and $0.602 \%$ in $\mathrm{B}-\mathrm{O}$ and $\mathrm{B}-\mathrm{B}$ bonds, respectively. That being said, boroxine can be used in high temperatures environment, such as space, aircraft, oil, and gas research applications. ${ }^{36}$ Nevertheless, it will be interesting to see the highest temperature range that $\mathrm{B}_{3} \mathrm{O}_{3}$ can withstand and, therefore, propose a separate investigation to find the melting point of $\mathrm{B}_{3} \mathrm{O}_{3}$.

\subsection{Mechanical properties}

In Fig. 6, we present the stress curve employed to calculate the in-plane elastic modulus $\left(Y_{2 \mathrm{D}}\right)$ of $2 \mathrm{D}$ boroxine. We vary the lattice constant in small steps and observe the energy change as
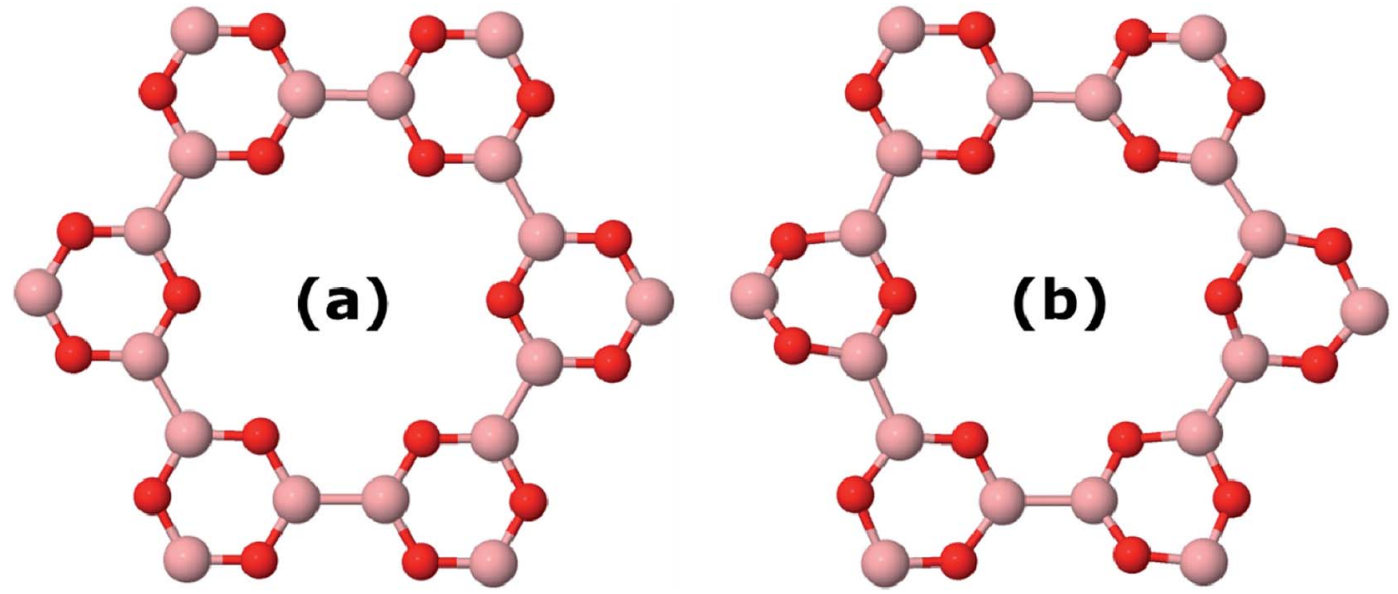

Fig. 5 Snapshots of the results of AIMD of boroxine at (a) $0 \mathrm{~K}$, and (b) $600 \mathrm{~K}$. The negligible distortion in the structure reflects its robustness. 


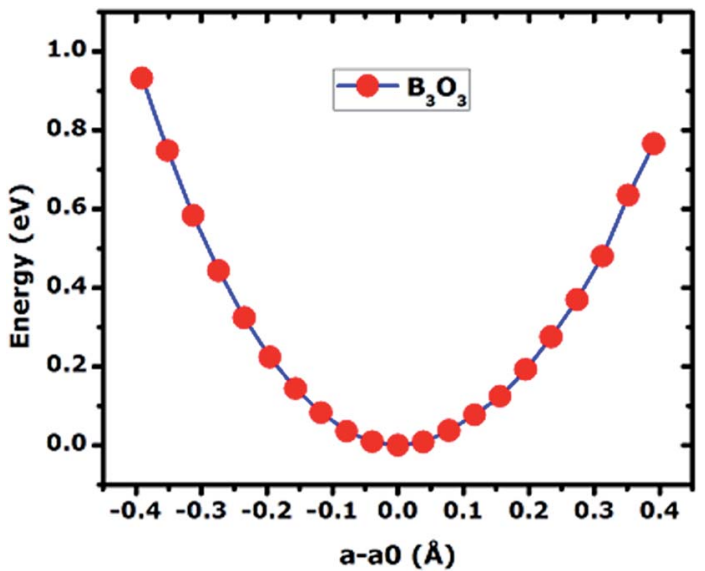

Fig. 6 The curve of total energy (in eV) vs. change in lattice constant (in $\AA$ ) calculated of $2 \mathrm{D} \mathrm{B}_{3} \mathrm{O}_{3}$ at the hybrid level of theory. The in-plane stiffness is calculated by taking the second derivative at the minimum.

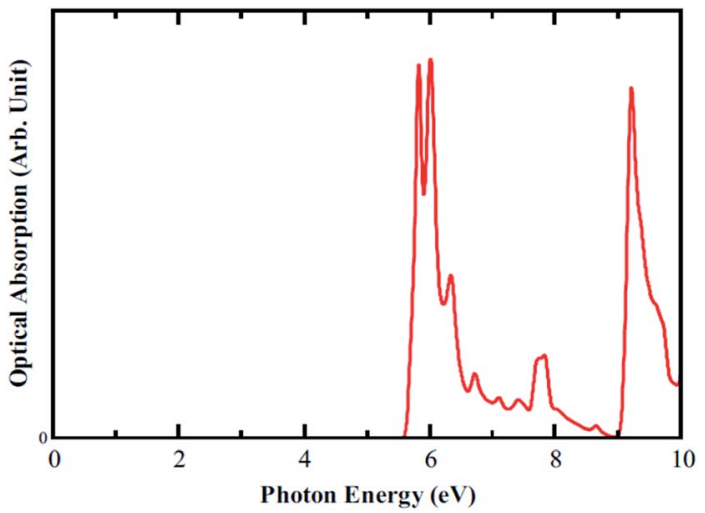

Fig. 7 Optical Absorption spectra calculated for $\mathrm{B}_{3} \mathrm{O}_{3}$, at the hybrid level of theory.

Table 1 Adsorption energies for lithium, sodium, and potassium at the M06-L/6-31G* level of theory

\begin{tabular}{lll}
\hline & Infinite boroxine & $\left(\mathrm{B}_{3} \mathrm{O}_{3}\right)_{6} \mathrm{H}_{6}$ flake \\
\hline $\mathrm{Li}$ & -52.0 & -55.2 \\
$\mathrm{Na}$ & -50.6 & -54.2 \\
$\mathrm{~K}$ & -64.1 & -67.8 \\
$\mathrm{Li}^{+}$ & & -60.7 \\
$\mathrm{Na}^{+}$ & & -51.63 \\
$\mathrm{~K}^{+}$ & & -47.3
\end{tabular}

a function of lattice parameter. The second derivative from the plot gives the $Y_{2 \mathrm{D}}$ and is given by: $Y_{2 \mathrm{D}}=\left.A_{0} \frac{\partial^{2} E}{\partial A^{2}}\right|_{A_{0}}=\left.\frac{\sqrt{ } 3}{6} \frac{\partial^{2} E}{\partial a^{2}}\right|_{a_{0}}, A=\frac{\sqrt{ } 3 a^{2}}{6}$, represents the $2 \mathrm{D}$ area of $\mathrm{B}_{3} \mathrm{O}_{3}$ and $a$ is the lattice constant. At the hybrid level, the 2D bulk modulus of $\mathrm{B}_{3} \mathrm{O}_{3}$ is $53.9 \mathrm{~N} \mathrm{~m}^{-1}$, about $17.93 \mathrm{~N} \mathrm{~m}^{-1}$ smaller than the values reported by Lin et $a .^{\mathbf{1 6}}$ The latter values are significantly smaller than the one determined for graphene $\left(206 \mathrm{~N} \mathrm{~m}^{-1}\right.$ ) in our earlier study at the same level of theory. ${ }^{31}$ The earlier reported value of $Y_{2 \mathrm{D}}$ of graphene $\left(206.6 \mathrm{~N} \mathrm{~m}^{-1}\right), \mathrm{hBN}$ $\left(177 \mathrm{~N} \mathrm{~m}^{-1}\right), \operatorname{SiC}\left(116.5 \mathrm{~N} \mathrm{~m}^{-1}\right)$, and $\mathrm{GeC}\left(101 \mathrm{~N} \mathrm{~m}^{-1}\right)$ are higher than that of $\mathrm{B}_{3} \mathrm{O}_{3}$. However, this value is slightly higher than that of $\mathrm{Be}_{3} \mathrm{~N}_{2}\left(46.5 \mathrm{~N} \mathrm{~m}^{-1}\right),{ }^{31}$ silicene $\left(44.5 \mathrm{~N} \mathrm{~m}^{-1}\right)$, and germanene $\left(29.6 \mathrm{~N} \mathrm{~m}^{-1}\right){ }^{37}$

\subsection{Optical properties}

Optical absorption spectra are also calculated for parallel polarization without taking into account the excitonic effect. The onset of optical absorption, which is the optical gap, appears around $5.7 \mathrm{eV}$ as can be seen in Fig. 7. This value is sufficiently larger than the electronic band gap of $3.66 \mathrm{eV}$, meaning that the optical transition from first valence to first conduction band is forbidden as per dipole selection rules. This can be understood by looking at the orbital character shown in PDOS plot. The first transition occurs from the blue peak to the green peak $\left(\mathrm{p}_{z}-\mathrm{p}_{z}\right)$. Moreover, the separation between these two pi bands is around $5.7 \mathrm{eV}$, thus agreeing well the optical gap. Additionally, the optical gap is in the deep UV range.

\subsection{Adsorption of alkalis}

As expressed in the first section, based on the chemistry displayed by the 18-crown-6 ether, we expect a strong affinity of $2 \mathrm{D}$ boroxine towards potassium. In Table 1, we list the adsorption energies computed for lithium, sodium, and potassium using finite and infinite models. The calculations were performed at the M06-L/6-31G* level of theory and we used a larger unit cell composed by six $\mathrm{B}_{3} \mathrm{O}_{3}$ rings so that the unit cell contains a complete hole (as shown in Fig. 1, but including the translucent atoms). The adsorption energies of lithium, sodium, and potassium on the hexagonal hole of boroxine are $-52.0,-50.6$ and $-64.1 \mathrm{kcal} \mathrm{mol}^{-1}$, respectively. Large differences, which may indicate that it is possible to separate selectively adsorb the latter alkali. However, for the adsorption of cations, the situation is reversed and lithium becomes clearly favored. In addition to this, it will also be interesting to integrate a metal atom in the hole of $\mathrm{B}_{3} \mathrm{O}_{3}$ and utilize it as a $\mathrm{B}_{3} \mathrm{O}_{3}$-supported metal single-atom catalyst, just like $\mathrm{C}_{2} \mathrm{~N}{ }^{38,39}$ With regard to the electronic properties, in Fig. 8 we present the band structure and total and projected density of states for boroxine with a potassium atom adsorbed in the hole. The band gap determined for boroxine with a $\mathrm{Li}, \mathrm{Na}$ and $\mathrm{K}$ atoms adsorbed in the hole are: $0.16 / 3.86,0.03 / 3.79$ and $0.08 / 3.93 \mathrm{eV}$, respectively, at the M06-L/ $6-31 G^{*}$ level of theory (spin-up/spin-down notation). Thus, alkali atom adsorption dramatically changes the gap for one spin channel while the other is minimally affected. Therefore, it may be possible to attain selective pins polarized transport using these elements.

\subsection{Bilayer boroxine}

We also calculate $\mathrm{B}_{3} \mathrm{O}_{3}$ bilayers in $\mathrm{AA}, \mathrm{AB}$, and $\mathrm{AB} 2$ stacking as shown in Fig. 9. In AA stacking, the interlayer distance is about $3.57 \AA$. In $\mathrm{AB}$ stacking, the second layer is translated about 1.39 $\AA$ (the $\mathrm{B}-\mathrm{O}$ bond length) along the $x$-axis, whereas, in AB2 stacking, this translation is about $2.78 \AA$ (the next neighboring 

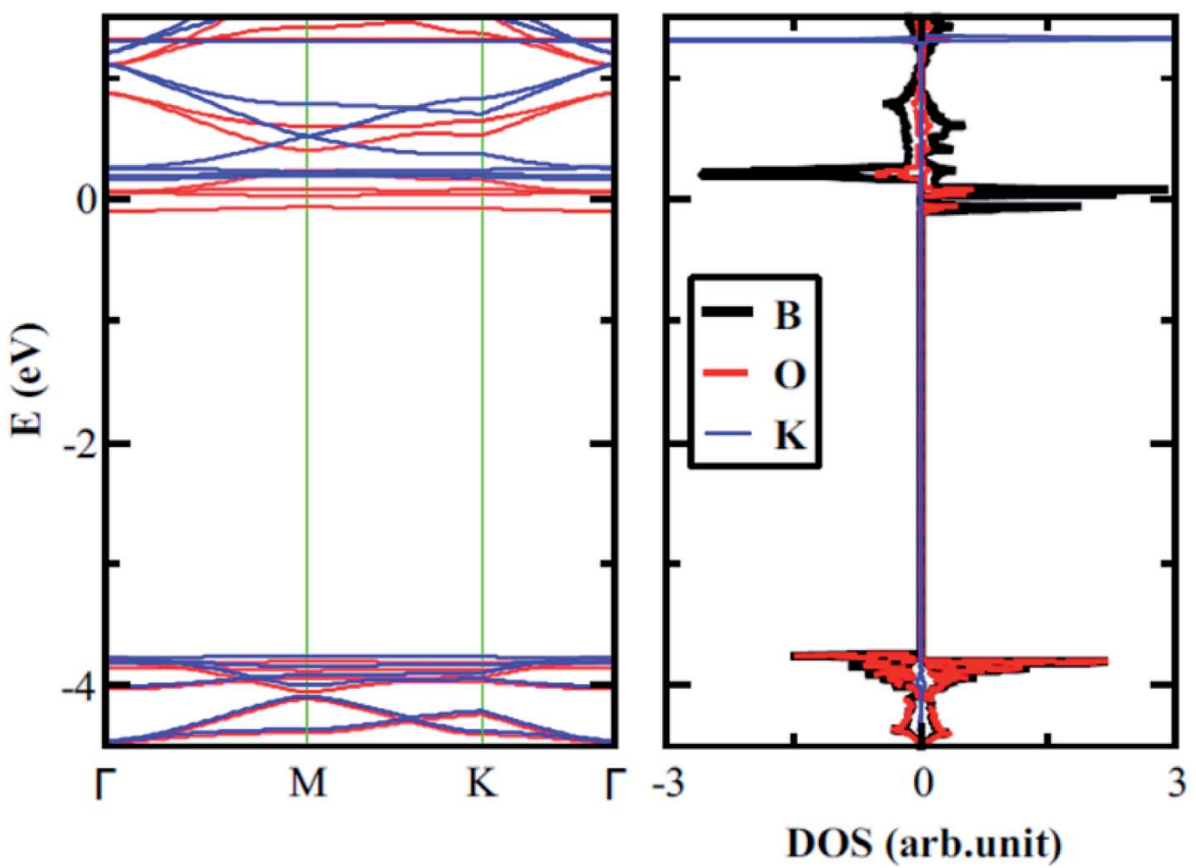

Fig. 8 Band structure and density of states determined for boroxine with a potassium atom adsorbed in the central hole, at the VDW-DF/DZP level of theory.

$\mathrm{B}-\mathrm{O}$ bond length) at PBE-D3 level. The interlayer separation in $\mathrm{AB}\left(h_{1}=3.18, h_{2}=3.3 \AA\right)$ is larger than that of $\mathrm{AB} 2\left(h_{1}=3.02, h_{2}\right.$ $=3.28 \AA$ ). Here, $h_{1}$ is the interlayer separation between $\mathrm{B}-\mathrm{O}$ (or $\mathrm{O}-\mathrm{B})$, whereas, $h_{2}$ refers to the separation between $\mathrm{B}-\mathrm{B}(\mathrm{O}-\mathrm{O})$ in $\mathrm{AB}$ ( $\mathrm{AB} 2$ ) bilayer. We show the periodic structures of these bilayers in Fig. S2 bilayer in ESI. $\dagger$ For comparison, the interlayer distance in bilayer graphene at PBE-D3 level is $3.33 \AA$, which is larger than that of AB2 stacking. Furthermore, the interlayer separation has a direct impact on the stability of the bilayers, $\mathrm{AB} 2$ being the most favorable, whereas, AA is the least. We also calculate the interlayer interaction energy (IIE) as: IIE $=\frac{2 E_{1}-E_{\text {tot }}}{N}$, where $E_{1}$ and $E_{\text {tot }}$ are the energies of

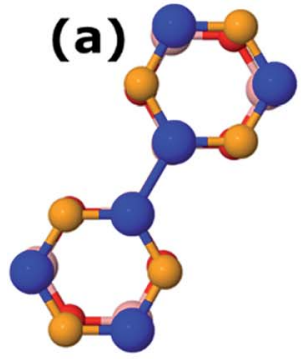

Boron-Layer2

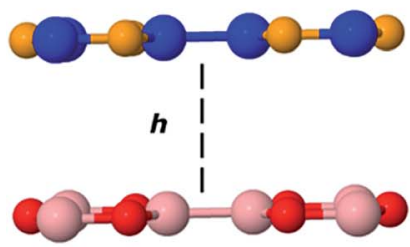

Boron-Layer1
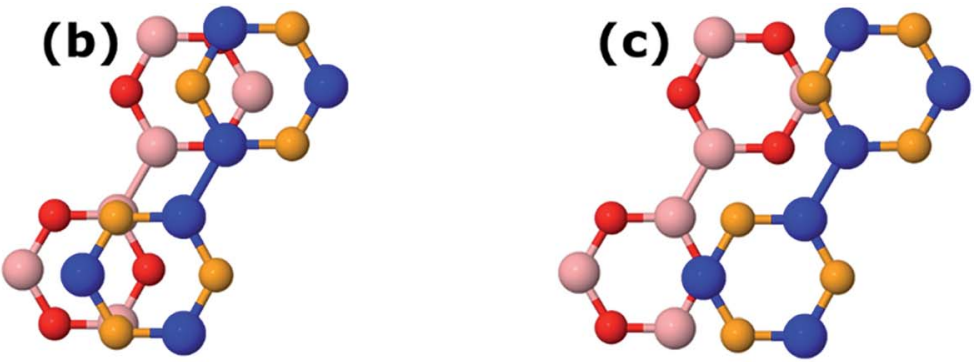

\section{Oxygen-Layer2}
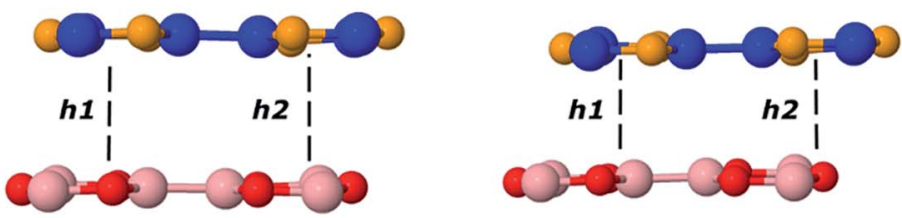

\section{Oxygen-Layer 1}

Fig. 9 Top and side view bilayer $B 303$ with (a) AA, (b) AB, and (c) AB2 stacking. In the AA pattern, $h$ represents the interlayer distance. In the other two stackings, $h_{1}$ and $h_{2}$ correspond to the separation between $\mathrm{B}-\mathrm{O}(\mathrm{O}-\mathrm{B})$ and $\mathrm{B}-\mathrm{B}(\mathrm{O}-\mathrm{B})$, respectively. 
Table 2 Relative energy (in meV), interlayer interaction energy (IIE) (in meV per atom), exfoliation energy ( $E_{\text {exf }}$ ) (in meV $A^{-2}$ ), and interlayer separation at $\mathrm{PBE}-\mathrm{D} 3$ level in $\mathrm{B}_{3} \mathrm{O}_{3}$ patterns. The $h$ represents interlayer distance in the AA pattern, whereas, $h_{1} / h_{2}$ represents $\mathrm{B}-\mathrm{O} / \mathrm{B}-\mathrm{B}$ (or $\mathrm{O}-\mathrm{O}$ ) separation in different layers of other patterns

\begin{tabular}{llll}
$\begin{array}{l}\text { Relative energy } \\
(\mathrm{meV})\end{array}$ & $\begin{array}{l}\text { IIE } \\
(\mathrm{meV} \text { per atom })\end{array}$ & $\begin{array}{l}E_{\text {exf }} \\
\left(\mathrm{meV} \mathrm{A}^{-2}\right)\end{array}$ & $\begin{array}{l}\text { Interlayer } \\
h, h_{1} / h_{2}(\AA)\end{array}$ \\
\hline 743.71 & & & 3.57 \\
589 & 6.45 & & $3.18 / 3.3$ \\
443 & 12.5 & & $3.02 / 3.28$ \\
358 & 16.05 & 2.8 & 3.48 \\
447 & 12.4 & 5.6 & $3.15 / 3.15$ \\
150 & 24.7 & 7 & $3.09 / 3.11$ \\
0 & 31 & & 3.33 \\
& 24 & 20 & 3.34
\end{tabular}

monolayer and bilayer, respectively. In addition, we compare the outcome with bilayer graphene. Interestingly, we find that IIE in AB2 stacking is comparable to that of bilayer graphene. Table 2 is decorated with relevant data at the PBE-D3 level. The results obtained using other methods are gathered in Table S2. $\dagger$ The electronic band structures are given in Fig. 10. Furthermore, the bilayer gaps are found to be lower than that of the single layer.

We also consider the effect of an external electric field on the electronic structure of $\mathrm{AB} 2$ stacked bilayer $\mathrm{B}_{3} \mathrm{O}_{3}$. We keep on increasing the field until we reach a limit where the gap becomes zero. The band structure plots are shown in Fig. S3 in ESI $\dagger$ and the band gap values as a function of the increasing field are shown in Fig. 11. We divide the plot into three parts where green, orange, and red colors represent reasonably-high, very-high, and extremely-high external electric field range. It is not known how much external electric field can be applied to

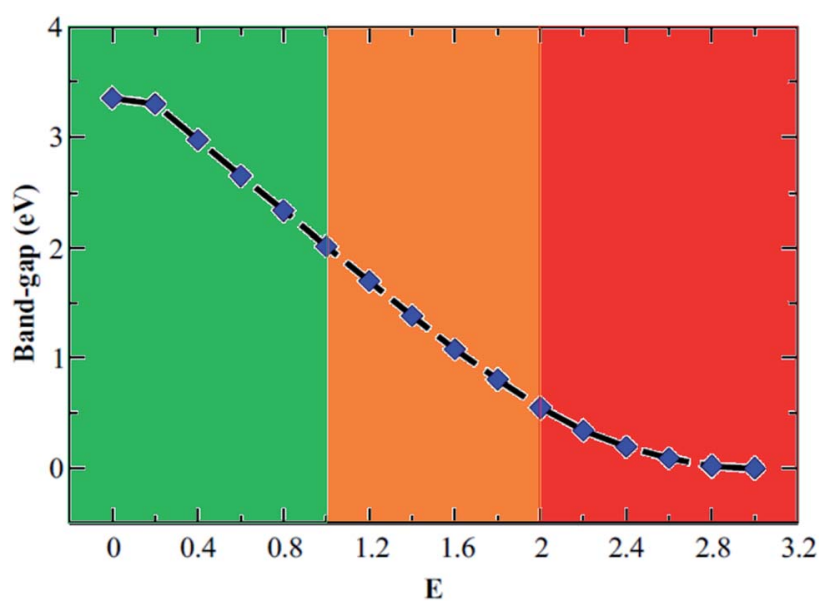

Fig. 11 Band gap (in eV) variation as a function of external electric field (in $\vee \AA^{-1}$ ) for $A B 2$ stacked bilayer boroxine.

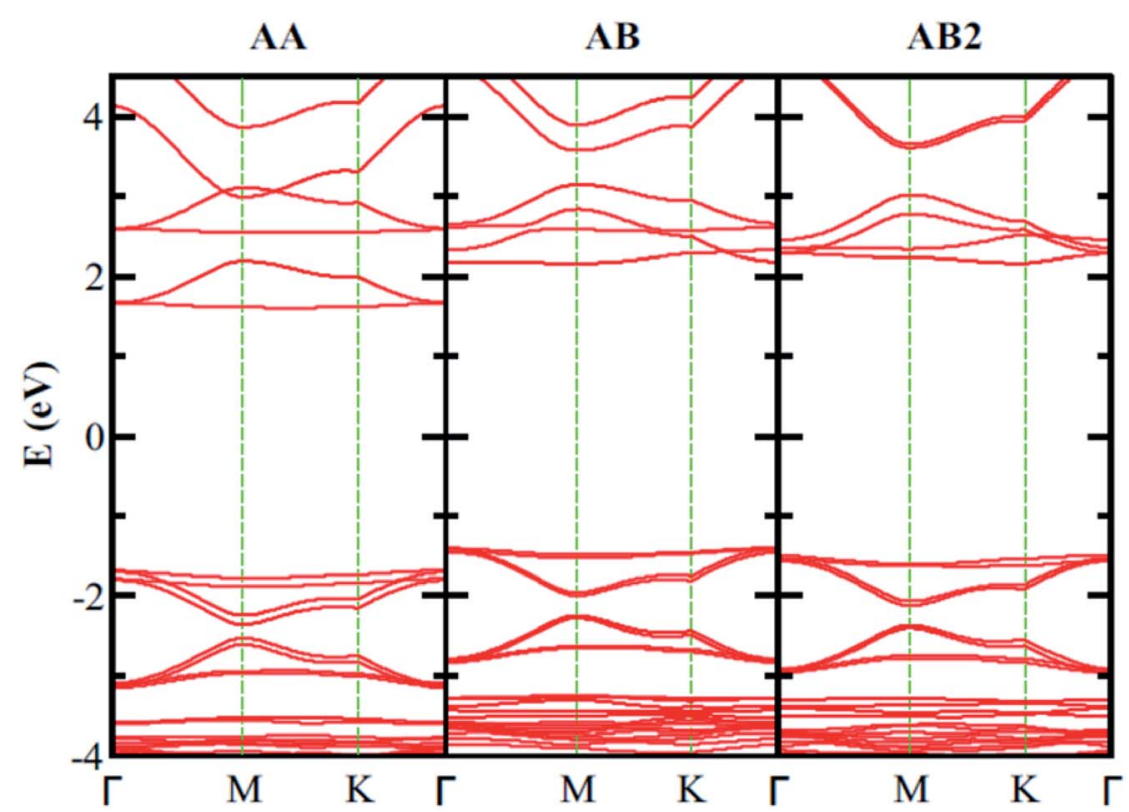

Fig. 10 Electronic band structure of bilayer $\mathrm{B}_{3} \mathrm{O}_{3}$ with different stacking at vdW-DF level. The band gaps in $A A, A B$, and $A B 2$ stacking are 3.28 eV, 3.56, and $3.65 \mathrm{eV}$, respectively, at the vdW-DF level. 

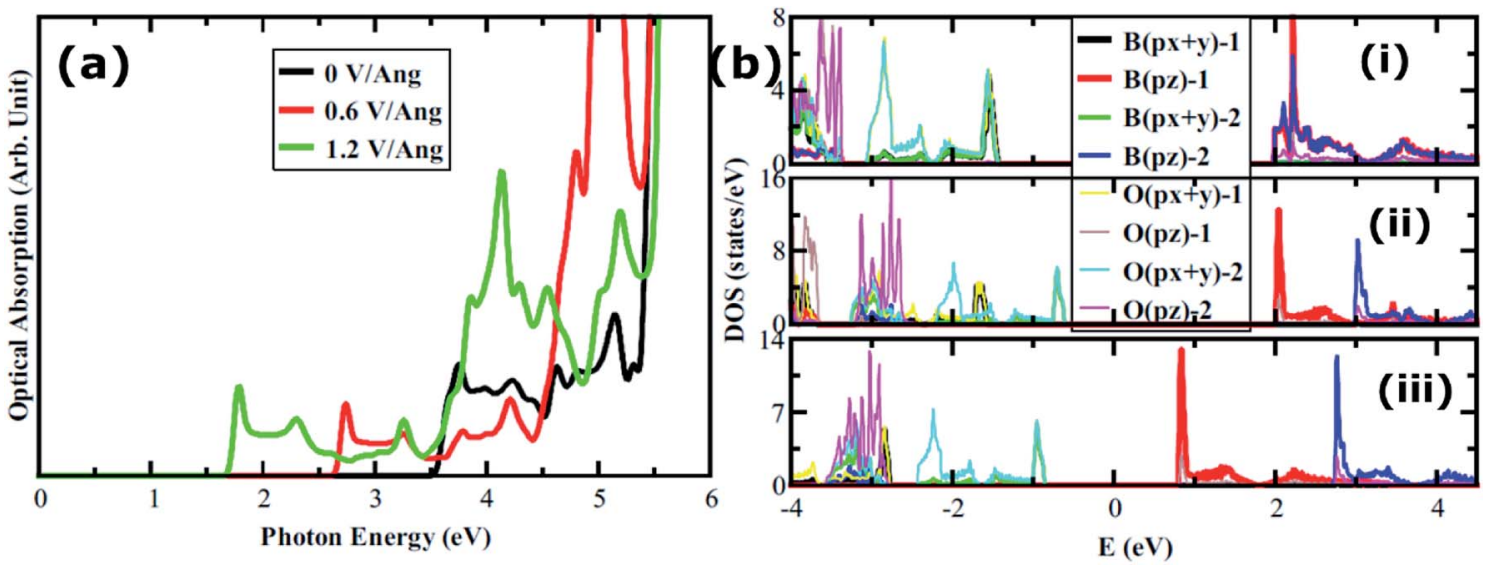

Fig. 12 (a) Optical absorption spectra of AB2 bilayer at various values of the external electric field. (b) Layer-resolved PDOS of AB2 bilayer (i) at 0 , (ii) 0.6 , and (iii) $1.2 \vee \AA^{-1}$.

$\mathrm{B}_{3} \mathrm{O}_{3}$ until it reaches its breakdown region. However, this study can motivate both theoreticians and experimentalists to initiate such an investigation. Moreover, it can be noted that the external electric field can significantly alter the electronic properties. The gap reaches a value of $1.7 \mathrm{eV}$ at a field of $1.2 \mathrm{~V}$ $\AA^{-1}$ from $3.47 \mathrm{eV}$ (at $0 \mathrm{~V} \AA^{-1}$ ). In addition to this, we also calculate the optical properties of AB2 bilayer with and without the external electric field. Here, we consider the structure at 0 , 0.6 , and $1.2 \mathrm{~V}^{-1}$ external electric field. The layer-resolved PDOS along with the optical absorption spectra is given in Fig. 12. Unlike the monolayer, it seems that the $\sigma$ to $\pi$ transition becomes allowed by the dipole selection rule in bilayer. Furthermore, there induce some extra peaks which seem to be coming from the transition between the broad $\mathrm{B}-\mathrm{O}-\sigma$ band and the narrow B-O- $\pi$ band. Most interestingly, these peaks correspond to interlayer transition and, furthermore, can be tuned right in the visible range. Nevertheless, those intense peaks in the deep UV range also look like the result of an interlayer transition. In photovoltaics, such transitions have an edge over the intralayer ones. ${ }^{\mathbf{4 0 - 4 4}}$ However, we propose that more accurate calculations, such as GW/BSE are required to confirm these outcomes.

\subsection{Graphite-like bulk B3O3}

Finally, we consider graphite-like $\mathrm{B}_{3} \mathrm{O}_{3}$ in bulk form which is a new phase of $\mathrm{B}_{3} \mathrm{O}_{3}$ at PBE-D3 level. We consider the same patterns as that of the bilayers. From the total energy calculations, it is evident that these patterns are local minima in the total energy surface, again AB2 pattern being the most favorable. The same outcome is confirmed by all the employed levels of theory. In bulk form, they exhibit the same band gaps like

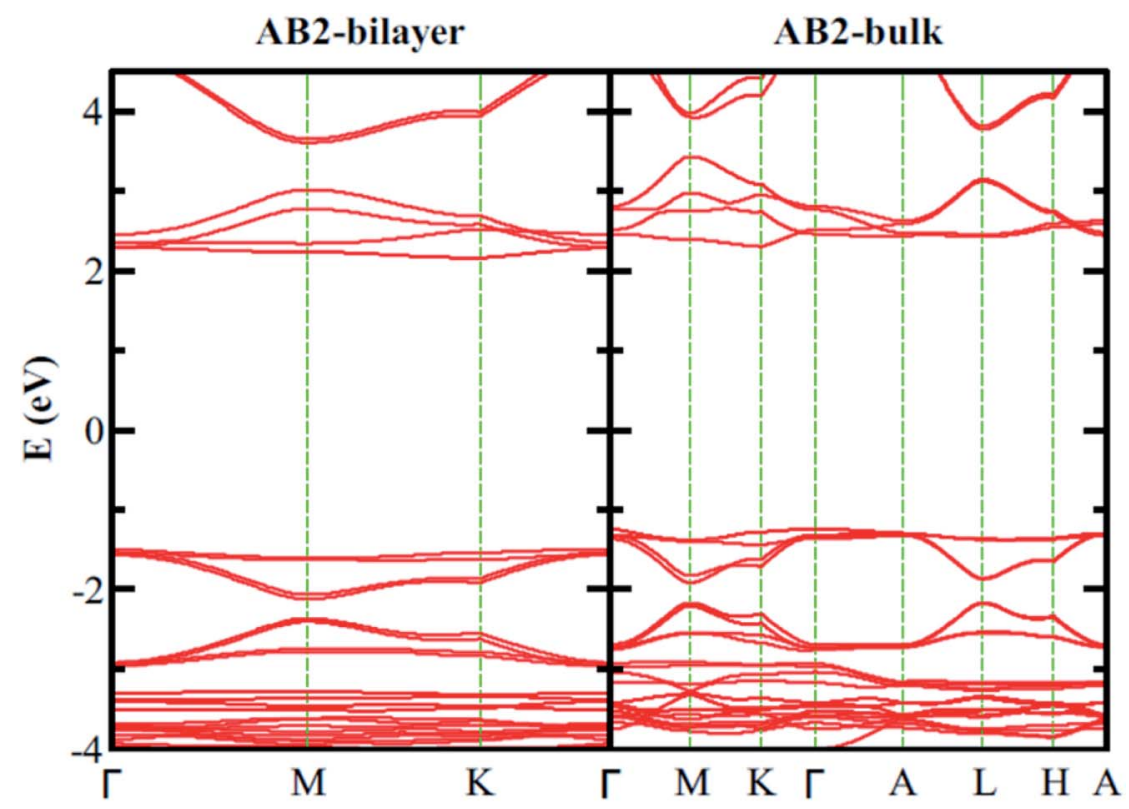

Fig. 13 Comparison of the electronic band structure of $A B 2$ stacking of $\mathrm{B}_{3} \mathrm{O}_{3}$ in bilayer and bulk form at vdW-DF level. 


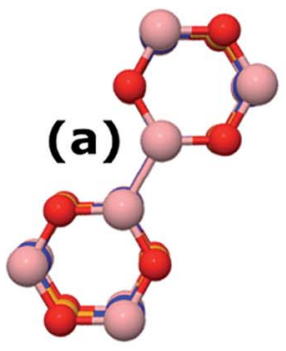

Boron-Layer2
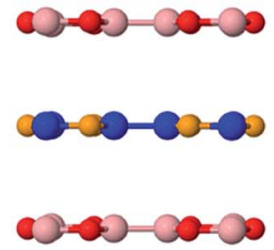

Boron-Layer1

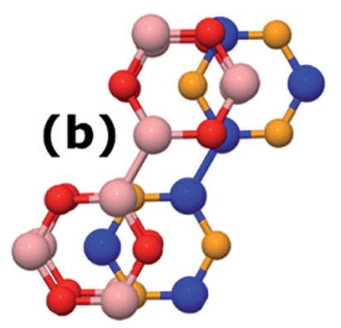

Oxygen-Layer 2
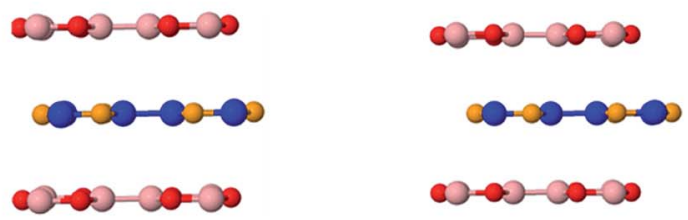

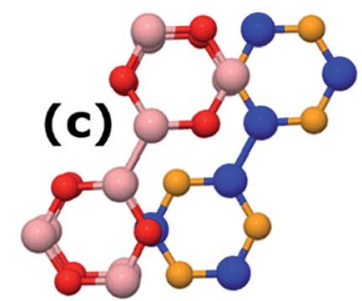

Oxygen-Layer 1

Fig. 14 Top and side view of (a) $A A$, (b) $A B$, and (c) $A B 2$ patterns of $\mathrm{B}_{3} \mathrm{O}_{3}$ in bulk form at PBE-D3 level.

that in the bilayer-form and as a comparison, the bilayer and bulk band structures of AB2 stacking are given in Fig. 13. We also calculate the exfoliation energy as reported elsewhere using the following equation: ${ }^{31} E_{\text {exf }}=\frac{E_{\mathrm{B}_{3} \mathrm{O}_{3}}-E_{\mathrm{g}-\mathrm{B}_{3} \mathrm{O}_{3}}}{A_{\mathrm{B}_{3} \mathrm{O}_{3}}}$, where the energy difference between monolayer $\mathrm{B}_{3} \mathrm{O}_{3}$ and graphite-like bulk $\mathrm{B}_{3} \mathrm{O}_{3}$ is divided by the monolayer area. From Table 2, it is evident that $\mathrm{B}_{3} \mathrm{O}_{3}$ in bulk form is not only comparably robust to that of graphite but the monolayer can be exfoliated with a lot ease as well. The geometries are given in Fig. 14 .

\section{Conclusions}

We employed first-principles calculations to perform a thorough theoretical characterization of the various aspects of the recently synthesized boroxine $\mathrm{B}_{3} \mathrm{O}_{3}$ monolayer. In agreement with previous theoretical investigations by Lin et al. ${ }^{16}$ we found that the structure is perfectly planar formed by $\mathrm{B}_{3} \mathrm{O}_{3}$ hexagons with hexagonal symmetry, joined by a strong covalent bond between $\mathrm{BB}$ as reveal by ELF. The six $\mathrm{B}_{3} \mathrm{O}_{3}$ units joined with sixfold symmetry exhibiting a large hole with a surface of $23 \AA^{2}$, which is ideal for the adsorption of potassium and the hydronium ion. In effect, we found that boroxine preferentially binds potassium over sodium. In contrast with previous works, we found that the band gap is direct. In effect, HSE calculations indicate that it is an insulator with a direct electronic band gap equal to $5.3 \mathrm{eV}$. Based on the cohesive energy calculations, $\mathrm{B}_{3} \mathrm{O}_{3}$ monolayer is comparably strong as that of graphene and remains negligibly distorted even at $600 \mathrm{~K}$ as from AIMD simulations. The elastic modulus is higher than that of $\mathrm{Be}_{3} \mathrm{~N}_{2}$, silicene, and germanene. Besides, we also explore the bilayer and bulk form of $\mathrm{B}_{3} \mathrm{O}_{3}$ and find that those phases are equally robust in comparison to bilayer graphene and graphite. Furthermore, the exfoliation energy calculations suggest that the exfoliation of monolayer $\mathrm{B}_{3} \mathrm{O}_{3}$ from the bulk phase is quite easier than that of graphene-graphite. In addition, we find a great tenability of the optoelectronic properties in the presence of external electric field. That being said, the optical properties can be tuned from deep UV to the visible range thus offering a vast range of applications in optoelectronics.

\section{Conflicts of interest}

The authors declare no conflicts of interest.

\section{References}

1 K. S. Novoselov, A. K. Geim, S. V. Morozov, D. Jiang, Y. Zhang, S. V. Dubonos, I. V. Grigorieva and A. A. Firsov, Science, 2004, 306, 666-669.

2 Z. Zeng, Z. Yin, X. Huang, H. Li, Q. He, G. Lu, F. Boey and H. Zhang, Angew. Chem., Int. Ed., 2011, 50, 11093-11097.

3 G. Ye, Y. Gong, J. Lin, B. Li, Y. He, S. T. Pantelides, W. Zhou, R. Vajtai and P. M. Ajayan, Nano Lett., 2016, 16, 1097-1103. 4 Y. Gogosti and B. Anasori, ACS Nano, 2019, 13, 8491-8494. 5 P. Vogt, P. De Padova, C. Quaresima, J. Avila, E. Frantzeskakis, M. C. Asensio, A. Resta, B. Ealet and G. Le Lay, Phys. Rev. Lett., 2012, 108, 155501.

6 P. A. Denis, Phys. Chem. Chem. Phys., 2015, 17, 5393-5402.

7 M. E. Davila, L. Xian, S. Cahangirov, A. Rubio and G. Le Lay, New J. Phys., 2014, 16, 095002.

8 J. Ji, X. Song, J. Liu, Z. Yan, C. Huo, S. Zhang, M. Su, L. Liao, W. Wang, Z. Ni, Y. Hao and H. Zeng, Nat. Commun., 2016, 7, 13352.

9 F. Reis, G. Li, L. Dudy, M. Bauernfeind, S. Glass, W. Hanke, R. Thomale, J. Schäfer and R. Claessen, Science, 2017, 357, 287-290. 
10 H. Liu, A. T. Neal, Z. Zhu, D. Tomanek and P. D. Ye, ACS Nano, 2014, 8, 4033.

11 Z. Zhu and D. Tomanek, Phys. Rev. Lett., 2012, 112, 176802.

12 M. Zhang, H. Sun, X. Wang, H. Du, J. He, Y. Long, Y. Zhang and C. Huang, J. Phys. Chem. C, 2019, 123, 5010-5016.

13 J. Wang, F. Ma and M. Sun, RSC Adv., 2017, 7, 16801-16822.

14 P. A. Denis and F. Iribarne, J. Phys. Chem. C, 2018, 122, 18583-18587.

15 M. Stredansky, A. Sala, T. Fontanot, R. Costantini, C. Africh, G. Comelli, L. Floreano, A. Morgante and A. Cossaro, Chem. Commun., 2018, 54, 3971.

16 S. Lin, J. Gu, H. Zhang, Y. Wang and Z. Chen, FlatChem, 2018, 9, 27-32.

17 M. Dion, H. Rydberg, E. Schroder, D. C. Langreth and B. I. Lundqvist, Phys. Rev. Lett., 2004, 92, 246401.

18 Y. Zhao and D. G. Truhlar, J. Chem. Phys., 2006, 125, 194101. 19 J. Heyd and G. E. Scuseria, J. Chem. Phys., 2004, 120, 72747280.

20 J. M. Soler, E. Aratacho, J. D. Gale, A. Garcia, J. Junquera, P. Ordejon and S.-D. Portal, The SIESTA Method for $A b$ Initio Order-N Materials Simulation, J. Phys.: Condens. Matter, 2002, 14, 2745-2779.

21 P. Ordejon, E. Artacho and J. M. Soler, Phys. Rev. B: Condens. Matter Mater. Phys., 1996, 53, R10441-R10444.

22 N. Troullier and J. L. Martins, Phys. Rev. B: Condens. Matter Mater. Phys., 1991, 43, 1993-2006.

23 M. J. Frisch, G. W. Trucks, H. B. Schlegel, G. E. Scuseria, M. A. Robb, J. R. Cheeseman, G. Scalmani, V. Barone, B. Mennucci, G. A. Petersson, H. Nakatsuji, M. Caricato, X. Li, H. P. Hratchian, A. F. Izmaylov, J. Bloino, G. Zheng, J. L. Sonnenberg, M. Hada, M. Ehara, K. Toyota, R. Fukuda, J. Hasegawa, M. Ishida, T. Nakajima, Y. Honda, O. Kitao, H. Nakai, T. Vreven, J. A. Montgomery, Jr., J. E. Peralta, F. Ogliaro, M. Bearpark, J. J. Heyd, E. Brothers, K. N. Kudin, V. N. Staroverov, R. Kobayashi, J. Normand, K. Raghavachari, A. Rendell, J. C. Burant, S. S. Iyengar, J. Tomasi, M. Cossi, N. Rega, J. M. Millam, M. Klene, J. E. Knox, J. B. Cross, V. Bakken, C. Adamo, J. Jaramillo, R. Gomperts, R. E. Stratmann, O. Yazyev, A. J. Austin, R. Cammi, C. Pomelli, J. W. Ochterski, R. L. Martin, K. Morokuma, V. G. Zakrzewski, G. A. Voth, P. Salvador, J. J. Dannenberg, S. Dapprich, A. D. Daniels, Ö. Farkas, J. B. Foresman, J. V. Ortiz, J. Cioslowski and D. J. Fox, Gaussian 09, Revision D.01, Gaussian, Inc., Wallingford CT, 2009.
24 W. Hehre, L. Radom, P. v. R. Schleyer, and J. A. Pople, $A b$ initio Molecular Orbital Theory, Wiley, New Work, 1986.

25 S. Grimme, J. Comput. Chem., 2006, 27, 1787-1799.

26 S. Grimme, S. Ehrlich and L. Goerigk, J. Comput. Chem., 2011, 32, 1456-1465.

27 G. Kresse and J. Hafner, Phys. Rev. B: Condens. Matter Mater. Phys., 1994, 49, 14251-14269.

28 J. P. Perdew, K. Burke and M. Ernzerhof, Phys. Rev. Lett., 1996, 77, 3865-3868.

29 J. P. Perdew, K. Burke and M. Ernzerhof, Phys. Rev. Lett., 1997, 78, 1396.

30 P. E. Blöchl, Phys. Rev. B: Condens. Matter Mater. Phys., 1994, 50, 17953-17979.

31 G. Kresse and J. Furthmuller, Comput. Mater. Sci., 1996, 6, 15. 32 S. Ullah, P. A. Denis, R. B. Capaz and F. Sato, New J. Chem., 2019, 43, 2933-2941.

33 C. A. Coulson and T. W. Dingle, Acta Crystallogr., Sect. B: Struct. Crystallogr. Cryst. Chem., 1968, 24, 153.

34 A. Savin, R. Nesper, S. Wengert and T. F. Fässler, Angew. Chem., Int. Ed., 1997, 36, 1808-1832.

35 D. Toffoli, M. Stredansky, Z. Feng, G. Balducci, S. Furlan, M. Stener, H. Ustunel, D. Cvetko, G. Kladnik, A. Morgante, A. Verdini, C. Dri, G. Comelli, G. Fronzoni and A. Cossaro, Chem. Sci., 2017, 8, 3789-3798.

36 J. Tersoff and D. R. Hamann, Phys. Rev. B: Condens. Matter Mater. Phys., 1985, 31, 805-813.

37 C. Buttay, D. Planson, B. Allard, D. Bergogne, P. Bevilacqua, C. Joubert, M. Lazar, C. Martin, H. Morel, D. Tournier and C. Raynaud, J. Mater. Sci. Eng. B, 2011, 176, 283-288.

38 R. C. Andrew, R. E. Mapasha, A. M. Ukpong and N. Chetty, Phys. Rev. B: Condens. Matter Mater. Phys., 2012, 85, 125428. 39 L. Liu and A. Corma, Chem. Rev., 2018, 118, 4981-5079.

40 J. Mahmood, F. Li, C. Kim, H.-J. Choi, O. Gwon, S.-M. Jung, J.-M. Seo, S.-J. Cho, Y.-W. Ju, H. Y. Jeong, G. Kim and J.-B. Baek, Nano Energy, 2018, 44, 304-310.

41 S. Ullah, P. A. Denis, M. G. Menezes and F. Sato, Appl. Surf. Sci., 2019, 493, 308-319.

42 S. Gélinas, O. Paré-Labrosse, C.-N. Brosseau, S. AlbertSeifried, C. R. McNeill, K. R. Kirov, I. A. Howard, R. Leonelli, R. H. Friend and C. Silva, J. Phys. Chem. C, 2011, 115, 7114-7119.

43 S. Gélinas, A. Rao, A. Kumar, S. L. Smith, A. W. Chin, J. Clark, T. S. van der Poll, G. C. Bazan and R. H. Friend, Science, 2014, 343, 512.

44 M. M. Furchi, A. Pospischil, F. Libisch, J. Burgdörfer and T. Mueller, Nano Lett., 2014, 14, 4785-4791. 